\title{
Application of an artificial neural network to typhoon rainfall forecasting
}

\author{
Gwo-Fong Lin* and Lu-Hsien Chen \\ Department of Civil Engineering, National Taiwan University, Taipei 10617, Taiwan
}

\begin{abstract}
:
A neural network with two hidden layers is developed to forecast typhoon rainfall. First, the model configuration is evaluated using eight typhoon characteristics. The forecasts for two typhoons based on only the typhoon characteristics are capable of showing the trend of rainfall when a typhoon is nearby. Furthermore, the influence of spatial rainfall information on rainfall forecasting is considered for improving the model design. A semivariogram is also applied to determine the required number of nearby rain gauges whose rainfall information will be used as input to the model. With the typhoon characteristics and the spatial rainfall information as input to the model, the forecasting model can produce reasonable forecasts. It is also found that too much spatial rainfall information cannot improve the generalization ability of the model, because the inclusion of irrelevant information adds noise to the network and undermines the performance of the network. Copyright (C) 2005 John Wiley \& Sons, Ltd.
\end{abstract}

KEY WORDS neural network; typhoon rainfall; forecast; semivariogram

\section{INTRODUCTION}

Rainfall is an important resource in the Taiwan area. However, among all weather-related disasters, rainfall also causes the most damage. The island of Taiwan is situated in one of the main paths of northwestern Pacific typhoons. On average, three to four typhoons attack the island each year. Typhoon rainfall can be a most valuable resource if good forecasts and proper disaster-mitigation measures are made. Otherwise, it can cause serious damage. Improving typhoon-rainfall forecasting is always an important task of flood management in Taiwan. However, in order to develop a physically based mathematical model for typhoon-rainfall forecasting, one must know the behaviour of the physical process of typhoon rainfall, which is not an easy task. Typhoon rainfall is one of the most difficult elements of the hydrologic cycle to forecast because of its tremendous variability over a wide range of scales, both in space and time. A typhoon brings abundant rainfall along the path of its movement. The most obvious typhoon characteristics affecting the typhoon rainfall include the latitude and longitude of the typhoon centre, the maximum wind speed near the centre, the atmospheric pressure of the centre, the radius of winds over $15 \mathrm{~m} \mathrm{~s}^{-1}$, the speed of the typhoon movement, the direction of the typhoon movement, and the distance of the typhoon centre from a rain gauge. These characteristics are too complex to model directly.

Regarding the forecasting of rainfall at a certain rain gauge, some relevant studies available in the literature are reviewed herein. Johnson and Bras (1980) forecast rainfall using radar storm-tracking signals. This approach has received increasing attention recently. However, direct use of radar rainfall data in hydrologic forecasting is not, in general, an acceptable practice because of the various sources of error associated with radar observation of rainfall (Smith et al., 1996). Georgakakos and Bras (1984a,b) predicted the precipitation rate using the surface pressure, temperature and dew-point temperature. Burlando et al. (1993) assumed

\footnotetext{
* Correspondence to: Gwo-Fong Lin, Department of Civil Engineering, National Taiwan University, Taipei 10617, Taiwan.
}

E-mail: gflin@ntu.edu.tw 
that hourly rainfall follows an autoregressive moving-average process and thereby forecast rainfall on this basis. Eltahir (1998a,b) performed an analysis on the correlation between soil moisture and precipitation and suggested the possibility of forecasting the precipitation using the correlation.

Artificial neural networks (ANNs) have found increasing applications in various aspects of hydrology because of their ability to model both linear and nonlinear systems without the need to make any of the assumptions that are implicit in most traditional statistical approaches. Previous studies have shown the potential of ANNs for modelling hydrological processes (Zhu and Fujita, 1994; Hsu et al., 1995; Minns and Hall, 1996; Govindaraju and Rao, 2000). The available rainfall forecasting models built by ANNs are based on training the current spatial rainfall information from nearby rain gauges in the study catchments (French et al., 1992; Luk et al., 2000). However, in addition to the spatial rainfall information, a great amount of relative information must be involved in developing a typhoon-rainfall forecasting model. Moreover, in the past, if one wanted to know how many nearby rain gauges are needed, there was a needed to make a comparison test on the forecast accuracy among the ANNs configured with different numbers of nearby rain gauges. For example, there are 10 rain gauges in the study area examined in this paper. If hourly rainfall records for all nearby rain gauges are used as inputs to the model to forecast the rainfall at a certain rain gauge, then the data from the other nine gauges will be added gradually to the network. Therefore, if there are many rain gauges in the study area, one has to spend a lot of time on choosing the number of nearby rain gauges for each rain gauge. Hence, it is justified to develop a model that can consider the other factors of influence besides the spatial rainfall information and to find a better method for determining the number of rain gauges required whose information will serve as input to the model.

In this paper, an ANN model is adopted to forecast short-term typhoon rainfall. First, the model configuration is evaluated using eight typhoon characteristics. Second, for each rain gauge a semivariogram is also applied to determine the number of nearby rain gauges whose rainfall information will be used as input to the model. Third, the forecasting model, with the typhoon characteristics and the spatial rainfall information as inputs, is applied to two typhoon events to find the 1 hour ahead forecasts of typhoon rainfall.

\section{METHODOLOGY}

\section{ANN approach}

Because the obvious factors having an influence on the typhoon rainfall at any given location are very complex, a neural network with two hidden layers is constructed herein. That is, the ANN has four layers: one input layer, two hidden layers and one output layer. The basic architecture of a four-layered neural network is shown in Figure 1. The processing elements in each layer are called nodes or units. The parameters associated with each of these connections are called weights. The network shown herein is fully connected. This means that a node in any layer of the network is connected to all the nodes in the previous layer.

The iterative process for determining appropriate weights, called neuro-learning, minimizes the objective function $F$ :

$$
F=\frac{1}{2}\left[\sum_{i=1}^{M} \sum_{j=1}^{N}\left(d_{i j}-y_{i j}\right)^{2}\right]
$$

where $d_{i j}$ is the desired output, $y_{i j}$ is the actual output, $M$ is the number of samples in the training data set and $N$ is the number of nodes in the output layer. Learning continues until $F$ converges to an acceptably small value.

Back-propagation is the most popular among the neuro-learning algorithms available for adjusting the weight coefficient $w_{i j}^{k-1, k}$ between node $i$ in layer $k-1$ and node $j$ in layer $k$. In back-propagation, learning 


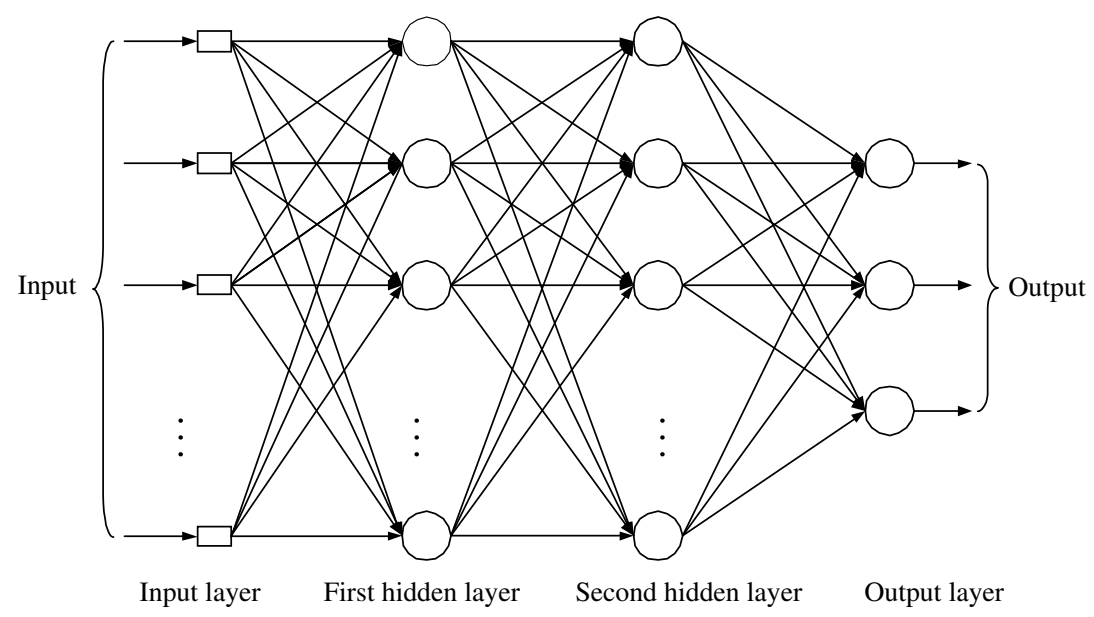

Figure 1. Architectural graph of a neural network with two hidden layers

proceeds from the output layer toward the input layer, i.e. in the direction opposite to signal propagation. The output $u_{j}^{k}$ from node $j$ in layer $k$ can be obtained from

$$
u_{j}^{k}=\sum_{i=1}^{m} w_{i j}^{k-1, k} u_{i}^{k-1}
$$

where $m$ is the total number of inputs applied to node $i$ in layer $k-1$. This method defines the change of weight with the progress of learning by

$$
\Delta w_{i j}^{k-1, k}(n+1)=\eta \delta_{j}^{k} u_{i}^{k-1}+\alpha \Delta w_{i j}^{k-1, k}(n)
$$

where $n$ is the iteration number, $u_{i}^{k-1}$ is the output from node $i$ in layer $k-1$, and $\delta_{j}^{k}$ is the local gradient for node $j$ in layer $k ; \eta$, the learning-rate parameter, and $\alpha$, the momentum constant, are positive constants. The $\delta_{j}^{k}$ term can be calculated by

$$
\delta_{j}^{k}= \begin{cases}e_{j} \frac{\mathrm{d} f\left(u_{j}^{l}\right)}{\mathrm{d} u_{j}^{l}} & \text { for node } j \text { in output layer } l \\ \frac{\mathrm{d} f\left(u_{j}^{k}\right)}{\mathrm{d} u_{j}^{k}} \sum_{r=1}^{m_{k+1}} \delta_{r}^{k+1} w_{j r}^{k, k+1} & \text { for node } j \text { in hidden layer } k\end{cases}
$$

where $f$ is the output function for each node, $m_{k+1}$ is the total number of nodes in layer $k+1$ which are connected to node $j$ in layer $k$, and $e_{j}$ is the error at the output of node $j$. The output function used most often for ANNs is the sigmoid function:

$$
f\left(u_{j}^{k}\right)=\frac{1}{1+\exp \left(-u_{j}^{k}\right)}
$$

in which $u_{j}^{k}$ can vary within the range $\pm \infty$, but $f\left(u_{j}^{k}\right)$ is bounded between zero and one.

The network topology directly affects its generalization capability and computational complexity. Therefore, determining an appropriate architecture of a neural network for a particular problem is an important issue. There is no established methodology for selecting the appropriate network architecture prior to training. In this paper, we resort to the trial-and-error method commonly used for network design. In addition, there is 
a distinct possibility that the model with the best-performing parameter values may end up overfitting the validation data. The network becomes well trained, but its generalization ability is not good. This means it does not learn enough about the past to generalize in the future. For this reason, cross-validation is used herein as a stopping criterion, for which training, validation and testing data sets are needed. The training data are used to find an optimal set of connecting weights, and the testing data are used to choose the best network configuration. Once an optimal network has been found, a validation is required in order to test the true generalization ability of the model. In the process of structuring the network, the early stopping technique is used in the following manner. Let $E_{\text {train }}(n)$ be the mean-square error per sample over the training data set after iteration $n$ and $E_{\text {valid }}(n)$ be the corresponding error on the validation data set. The $E_{\text {train }}(n)$ decreases as the training process continues. When $E_{\text {valid }}(n)$ starts to increase, then the training of the network is stopped. Using the early stopping technique can reduce the training time. Hence, the model can be retrained on-line to adapt to changing future events.

In this paper, using the present and past information of influence factors, the rainfall forecast at time $t+1$ at a rain gauge can be formulated as

$$
r(t+1)=f[x(t), x(t-1), x(t-2), \ldots, x(t-k+1)]
$$

where $t$ is the time, $x(t)=\left[x_{1}, x_{2}, \ldots, x_{n}\right]^{\mathrm{T}}$ represents a vector of influence factors at time $t, r(t+1)$ is the rainfall depth at time $t+1$, and $f[]$ is a nonlinear function that can be approximated using the ANN. The model with parameter $k$ in Equation (6) is termed the 'lag- $k$ model' herein. The lag-1 model considers only $x(t)$, the present information of influencing factors. If $x(t)$ and $x(t-1)$ are both considered, then the model is referred to as the lag-2 model. In a like manner, the lag-3 model takes account of $x(t), x(t-1)$ and $x(t-2)$.

\section{Semivariogram method}

The semivariogram represents the variation of the semivariance as a function of the horizontal distance and is employed to determine the optimal weights used with kriging methods. In the process of kriging analysis, the semivariogram plays a central role in the analysis of geostatistical data. A measure relating to the length of influence of a variable is termed the range. Within this range, sample points are related to each other, and all known sample points contained in this region must be considered when an unknown point of interest is estimated. Therefore, the semivariogram method can be applied as a tool to determine the number of spatial inputs.

The experimental semivariogram $\hat{\gamma}(h)$ is computed as half the average squared difference between the components of data pairs:

$$
\hat{\gamma}(h)=\frac{1}{2 N(h)} \sum_{i=1}^{N(h)}\left[z\left(s_{i}\right)-z\left(s_{i}+h\right)\right]^{2}
$$

where $N(h)$ is the number of pairs of observations separated by distance $h$, and $z\left(s_{i}\right)$ and $z\left(s_{i}+h\right)$ are the sample values of the variable $z$ at locations $s_{i}$ and $s_{i}+h$. There are several theoretical semivariogram models, e.g. Gaussian, spherical and exponential. Because the exponential model is versatile and has a simple analytical form, it is a popular model in hydrologic applications (Kitanidis, 1993). The form of the exponential semivariogram model is

$$
\gamma(h)=\sigma^{2}\left[1-\exp \left(-\frac{h}{a}\right)\right]
$$

where $\sigma^{2}$ is the sill (variance) and $a$ is the scale parameter. It should be noted that the range is $\alpha \approx 3 a$ (Kitanidis, 1993). 
In this paper, the semivariogram with a lag time of $t$ is considered. According to Equation (7), the experimental semivariogram with a lag time of $h_{t}$ can be written as

$$
\hat{\gamma}\left(h_{s}, h_{t}\right)=\frac{1}{2 N\left(h_{s}\right)} \sum_{i=1}^{N\left(h_{s}\right)}\left[z\left(s_{i}+h_{s}, t+h_{t}\right)-z\left(s_{i}, t\right)\right]^{2}
$$

where $z\left(s_{i}, t\right)$ is the sample value of the variable $z$ at location $s_{i}$ at time $t, z\left(s_{i}+h_{s}, t+h_{t}\right)$ is the sample value of the variable $z$ at location $s_{i}+h_{s}$ at time $t+h_{t}$, and $N\left(h_{s}\right)$ is the number of pairs of observations separated by distance $h_{s}$.

\section{APPLICATION}

\section{The study area and data}

In this paper, the actual rainfall data from 10 rain gauges in the Tanshui River basin in northern Taiwan are used. The average slope of the Tanshui River is $1 / 45$, and the total area of the basin is $2726 \mathrm{~km}^{2}$. In this watershed, floods and debris flows caused by heavy typhoon rainfall are serious natural hazards. Reliable forecasts of typhoon rainfall can extend the time for issuing warnings and allow sufficient time for people in threatened areas to take appropriate actions. Hence, typhoon-rainfall forecasting is an important task in the study area.

The rainfall data are collected from computer archives of the Central Weather Bureau and the Water Resources Agency. Figure 2 shows the study area and the locations of 10 rain gauges. Rainfall records at $1 \mathrm{~h}$ intervals for the 10 rain gauges are available from 1980 to 1998. From these records, a total of 32 typhoon rainfall events are selected. The typhoon characteristics of these 32 events are used to develop the forecasting model. Of these 32 typhoon rainfall events, 24 events are selected as training set, six events are taken as the validation set, and the remaining two events are used as a testing set for testing the forecasting performance of the ANN model. The two typhoon events for testing are Typhoon Herb on 30 July 1996 and Typhoon Nelson on 21 August 1985.

\section{Training and testing details}

In our ANN model, there are 10 and 5 nodes in the first and second hidden layers respectively. For the data set used in the present study, the input variables and the target variables are first normalized linearly in the range of 0.0 to 1.0 . This range is selected because the activation function is bounded between 0.0 and 1.0. The normalization is performed using

$$
x_{\text {norm }}=\frac{x-x_{\min }}{x_{\max }-x_{\min }}
$$

where $x_{\text {norm }}$ is the normalized value, and $x_{\min }$ and $x_{\max }$ are respectively the minimum and maximum values in the data set. The weights of the network are initialized with normally distributed random numbers in the range of -1 to +1 . The learning-rate parameter $\eta$ and the momentum constant $\alpha$ are set at 0.01 and 0.5 respectively. Two stop criteria are adopted: the cross-validation and the fixed number of iterations. The maximum number of iterations is set at 10000 .

\section{Model development procedures}

The primary objective of this paper is to investigate the effects of the typhoon characteristics and the spatial rainfall information on short-term typhoon-rainfall forecasting. First, the model configuration is evaluated using eight typhoon characteristics, i.e. the latitude and longitude (degrees) of the typhoon centre, the maximum wind speed $\left(\mathrm{m} \mathrm{s}^{-1}\right)$ near the centre, the atmospheric pressure $(\mathrm{hPa})$ of the centre, the radius $(\mathrm{km})$ of winds over 

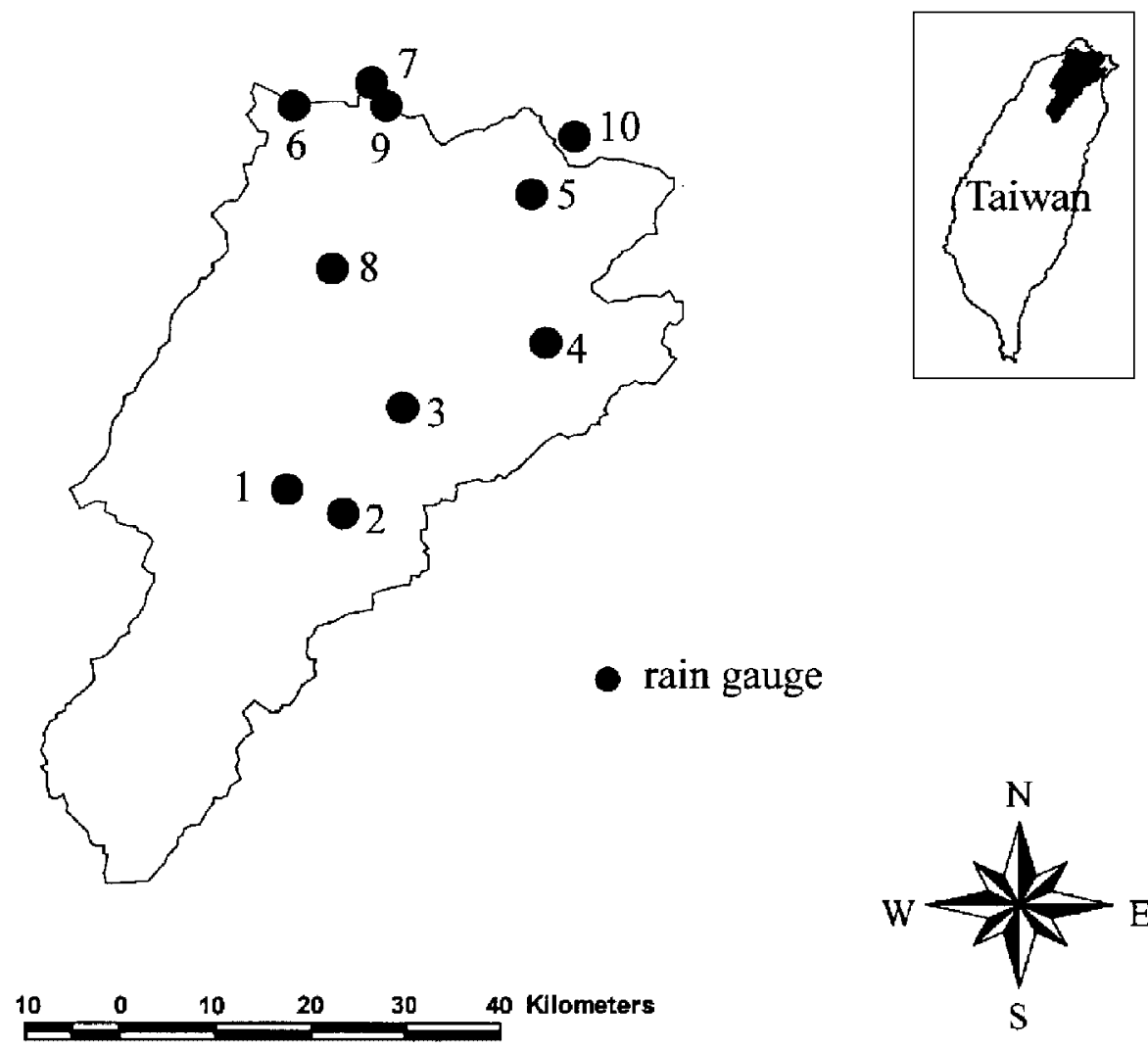

Figure 2. Locations of rain gauges in the Tanshui River basin

$15 \mathrm{~m} \mathrm{~s}^{-1}$, the speed $\left(\mathrm{km} \mathrm{h}^{-1}\right)$ of the typhoon movement, the direction (degrees) of the typhoon movement, and the distance $(\mathrm{km})$ of the typhoon centre from a rain gauge. Then different values of parameter $k$ in Equation (6) are tried to arrive at the optimal model. It should be noted that each rain gauge has its own $k$ value. The optimal model obtained in this manner for each rain gauge is referred to as ANN1, which uses only the information of typhoon characteristics as input.

As a next step in improving the model design, the influence of nearby rain gauges on the rainfall forecast at any given location in the study area is considered. The semivariogram with a lag time is also applied to determine the number of nearby rain gauges whose rainfall information will be used as input to the model. The number of nearby rain gauges required is different for each gauge. Using such spatial rainfall information and the typhoon characteristics, one can obtain a forecasting model, termed ANN2, for each rain gauge. Furthermore, the model that considers the rainfall information of 'all' rain gauges and the typhoon characteristics is also introduced. Such a model for each rain gauge is referred to as ANN3 herein.

\section{Criteria for evaluating model performance}

Three criteria that are commonly used to evaluate model performance are employed herein:

1. Root mean square error (RMSE)

$$
\operatorname{RMSE}=\sqrt{\frac{1}{n} \sum_{t=1}^{n}\left(\hat{R}_{t}-R_{t}\right)^{2}}
$$


where $\hat{R}_{t}$ is the predicted rainfall at time $t, R_{t}$ is the observed rainfall at time $t$, and $n$ is the number of time steps.

2. Coefficient of correlation (CC)

$$
\mathrm{CC}=\frac{\sum_{t=1}^{n}\left(R_{t}-\bar{R}\right)\left(R_{t}-\overline{\hat{R}}\right)}{\sqrt{\sum_{t=1}^{n}\left(R_{t}-\bar{R}\right)^{2} \sum_{t=1}^{n}\left(\hat{R}_{t}-\overline{\hat{R}}\right)^{2}}}
$$

where $\overline{\hat{R}}$ is the average of forecast rainfall and $\bar{R}$ is the average of observed rainfall.

3. Coefficient of efficiency (CE)

$$
\mathrm{CE}=1-\frac{\sum_{t=1}^{n}\left(R_{t}-\hat{R}_{t}\right)^{2}}{\sum_{t=1}^{n}\left(R_{t}-\bar{R}\right)^{2}}
$$

\section{RESULTS AND DISCUSSION}

First, we try to find the ANN1 for each rain gauge. ANN1 uses only the information of typhoon characteristics as input. Initially, the ANN1-lag-1 $\mathrm{h}$ model is developed. That is, the eight typhoon characteristics at time $t$ are used as inputs to the model and the output layer consists of a single unit representing the rainfall depth at time $t+1$. Therefore, the input layer and the output layer consist of eight units and one unit respectively. The ANN1-lag-2 $\mathrm{h}$ and ANN1-lag-3 $\mathrm{h}$ models are also tried to find the optimal model. Table I summarizes the performance of the three ANN1 models for 10 rain gauges. As shown in Table I, the ANN1-lag-2 h model is the best for each rain gauge. These results might indicate that the typhoon characteristics available in this study do not have a long-term memory property.

After the ANN1 (i.e. ANN1-lag-2 h) is developed for each rain gauge, one can forecast the rainfall at each rain gauge. The comparisons of observed and forecast hyetographs for Typhoon Herb are shown in Figure 3. Figure 3 shows that the forecasts for Typhoon Herb based on the typhoon characteristics are capable of showing the trend of rainfall when a typhoon is nearby. However, the method underestimates the rainfall

\begin{tabular}{|c|c|c|c|}
\hline \multirow[t]{2}{*}{ Station } & \multicolumn{3}{|c|}{$\mathrm{CE}$ for validation data } \\
\hline & Lag $1 \mathrm{~h}$ & Lag $2 \mathrm{~h}$ & Lag $3 \mathrm{~h}$ \\
\hline 1 & $0 \cdot 246$ & $0 \cdot 307$ & $0 \cdot 257$ \\
\hline 2 & $0 \cdot 178$ & $0 \cdot 217$ & $0 \cdot 182$ \\
\hline 3 & 0.195 & $0 \cdot 206$ & 0.174 \\
\hline 4 & $0 \cdot 204$ & $0 \cdot 218$ & $0 \cdot 190$ \\
\hline 5 & $0 \cdot 246$ & $0 \cdot 279$ & $0 \cdot 157$ \\
\hline 6 & $0 \cdot 203$ & $0 \cdot 223$ & $0 \cdot 143$ \\
\hline 7 & $0 \cdot 261$ & $0 \cdot 303$ & $0 \cdot 138$ \\
\hline 8 & $0 \cdot 111$ & $0 \cdot 231$ & $0 \cdot 159$ \\
\hline 9 & 0.238 & $0 \cdot 278$ & 0.239 \\
\hline 10 & $0 \cdot 159$ & $0 \cdot 186$ & $0 \cdot 146$ \\
\hline
\end{tabular}

Table I. Comparison of model lag for ANN1 

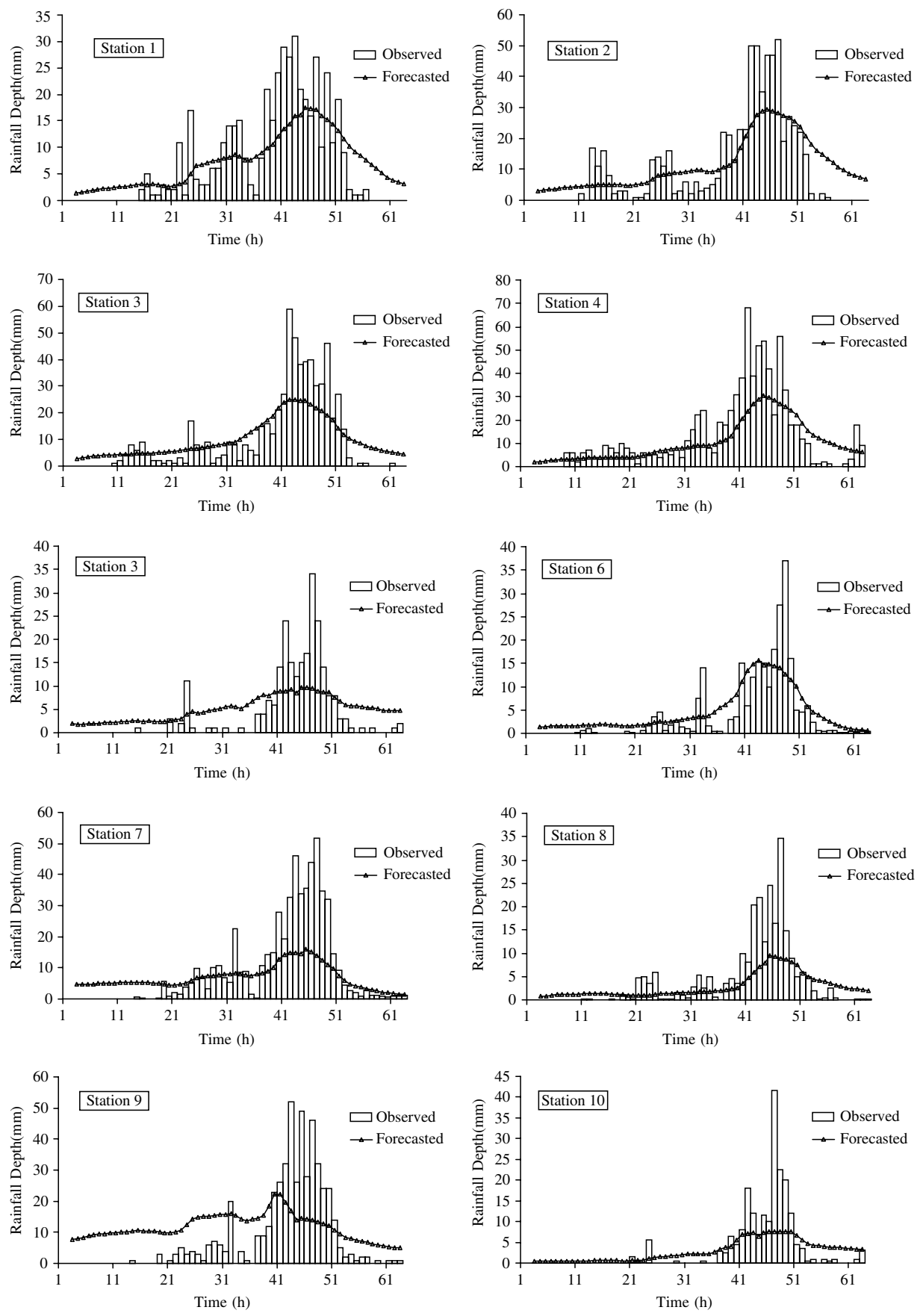

Figure 3. Comparisons of observed and forecast hyetographs for Typhoon Herb. Only the typhoon characteristics are used as input to the model 
depth for all gauges. For another testing event, Typhoon Nelson, the forecast hyetographs also have similar results.

ANN2 uses the typhoon characteristics and the optimal spatial rainfall information as inputs. Hence, one first has to determine the number of nearby rain gauges whose rainfall information will be used as input to the model. The fitted and experimental semivariograms with lag times of $1 \mathrm{~h}$ and $2 \mathrm{~h}$ based on the 30 rainfall events are shown in Figures 4 and 5 respectively. Figure 5 shows that the spatial rainfall correlation with a lag time of $2 \mathrm{~h}$ is low. Hence, the semivariogram with a lag time of $1 \mathrm{~h}$ is applied to determine the required number of nearby rain gauges. According to Figure 4, the range is equal to $33 \mathrm{~km}$, i.e. the rainfall records of rain gauges within this range will be chosen as the spatial input. For the 10 rain gauges in the study area, the

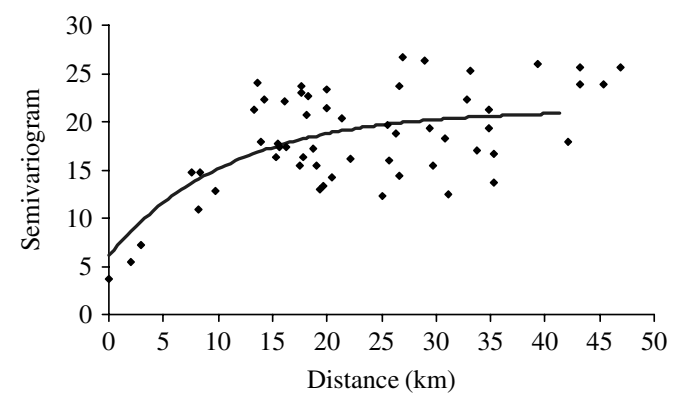

Figure 4. The fitted and experimental semivariograms with a lag time of $1 \mathrm{~h}$ for the 30 typhoon rainfall events

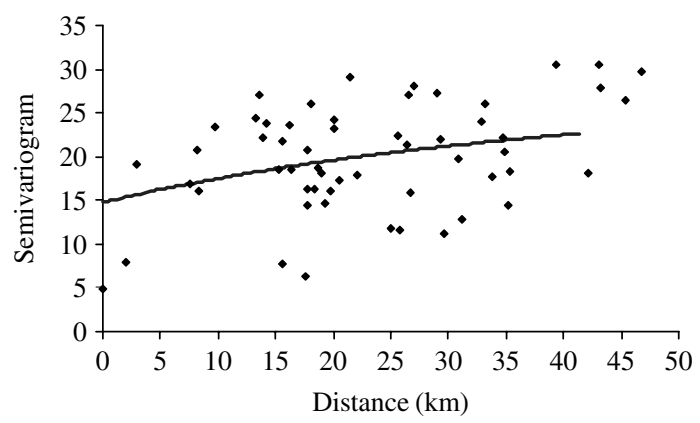

Figure 5. The fitted and experimental semivariograms with a lag time of $2 \mathrm{~h}$ for the 30 typhoon rainfall events

Table II. The number of rain gauges within the range

\begin{tabular}{cc}
\hline Station & No. of rain gauges \\
\hline 1 & 4 \\
2 & 4 \\
3 & 4 \\
4 & 5 \\
5 & 5 \\
6 & 4 \\
7 & 5 \\
8 & 7 \\
9 & 5 \\
10 & 3 \\
\hline
\end{tabular}



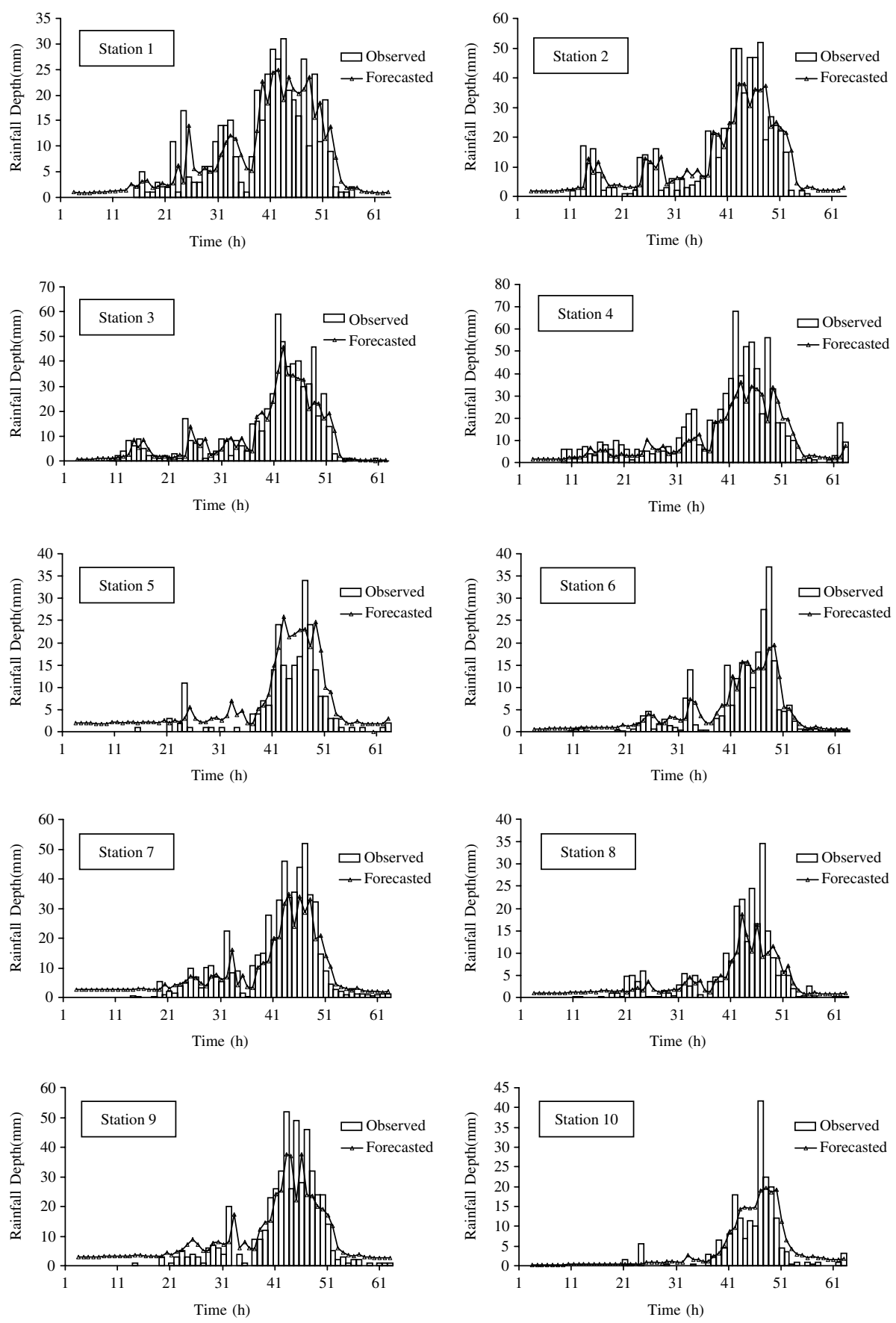

Figure 6. Comparisons of observed and forecast hyetographs for Typhoon Herb. The typhoon characteristics and the spatial rainfall information are used as inputs to the model 
corresponding numbers of rain gauges within the range are summarized in Table II. As shown in Table II, the required numbers of rain gauges are about half the number of all rain gauges in the study area.

The comparisons of observed and forecast hyetographs obtained by ANN2 for Typhoon Herb are shown in Figure 6. The results imply that ANN2 supports reasonable rainfall forecasting. For Typhoon Herb, the performance of ANN2 is summarized in Table III. ANN2 achieves RMSE values ranging from 3.83 to 8.61. The CCs are very high for 10 rain gauges. The CEs are all larger than 0.6 for all rain gauges. The forecasting results for Typhoon Herb indicate that ANN2 can forecast the hyetographs well. The RMSE, CC and CE from ANN1 are also presented in Table III for comparisons. Table III shows that ANN2 reduces the values of RMSE and raises the values of CC and CE compared with ANN1. These results indicate that the spatial input can increase the efficiency of the forecasting model. In a like manner, the RMSEs, CCs and CEs from ANN1 and ANN2 for Typhoon Nelson are summarized in Table IV. Again, ANN2 produces a better performance than ANN1.

Table V presents the CEs resulting from ANN2 and ANN3 for validation data. It should be noted that ANN3 uses the typhoon characteristics and all the spatial rainfall information as inputs. As shown in Table V, the model that considers the rainfall information of 'all' rain gauges does not perform well. In other words, ANN2 produces the most accurate rainfall among ANN1, ANN2 and ANN3. This is because the inclusion of irrelevant information adds noise to the network and undermines the performance of the network.

Table III. RMSE, CC and CE from ANN1 and ANN2 for Typhoon Herb

\begin{tabular}{|c|c|c|c|c|c|c|}
\hline \multirow[t]{2}{*}{ Station } & \multicolumn{2}{|c|}{ RMSE (mm) } & \multicolumn{2}{|c|}{$\mathrm{CC}$} & \multicolumn{2}{|c|}{$\mathrm{CE}$} \\
\hline & ANN1 & ANN2 & ANN1 & ANN2 & ANN1 & ANN2 \\
\hline 1 & $5 \cdot 88$ & 4.82 & 0.80 & 0.84 & 0.57 & 0.71 \\
\hline 2 & $9 \cdot 19$ & $7 \cdot 14$ & 0.81 & 0.88 & 0.59 & 0.75 \\
\hline 3 & 8.68 & $6 \cdot 02$ & 0.86 & 0.91 & 0.62 & 0.82 \\
\hline 4 & $10 \cdot 41$ & 8.61 & 0.82 & 0.84 & 0.56 & 0.62 \\
\hline 5 & $5 \cdot 64$ & $4 \cdot 16$ & 0.73 & 0.87 & $0 \cdot 36$ & 0.65 \\
\hline 6 & 4.62 & $3 \cdot 83$ & 0.78 & 0.86 & 0.58 & 0.71 \\
\hline 7 & $10 \cdot 25$ & $6 \cdot 17$ & 0.89 & 0.92 & $0 \cdot 39$ & 0.78 \\
\hline 8 & $5 \cdot 26$ & 4.40 & 0.80 & 0.80 & 0.42 & $0 \cdot 61$ \\
\hline 9 & 11.75 & 6.83 & 0.56 & 0.87 & $0 \cdot 18$ & 0.72 \\
\hline 10 & $5 \cdot 60$ & $3 \cdot 84$ & 0.72 & 0.84 & $0 \cdot 38$ & 0.71 \\
\hline
\end{tabular}

Table IV. RMSE, CC and CE from ANN1 and ANN2 for Typhoon Nelson

\begin{tabular}{|c|c|c|c|c|c|c|}
\hline \multirow[t]{2}{*}{ Station } & \multicolumn{2}{|c|}{ RMSE (mm) } & \multicolumn{2}{|c|}{$\mathrm{CC}$} & \multicolumn{2}{|c|}{$\mathrm{CE}$} \\
\hline & ANN1 & ANN2 & ANN1 & ANN2 & ANN1 & ANN2 \\
\hline 1 & 5.62 & 4.90 & 0.68 & 0.77 & 0.40 & 0.54 \\
\hline 2 & 11.89 & $7 \cdot 17$ & 0.77 & 0.91 & $0 \cdot 38$ & 0.77 \\
\hline 3 & 5.68 & 4.91 & 0.77 & 0.79 & 0.46 & 0.62 \\
\hline 4 & $8 \cdot 38$ & 6.46 & 0.63 & 0.77 & $0 \cdot 32$ & 0.61 \\
\hline 5 & 4.72 & 3.60 & 0.78 & 0.85 & 0.36 & 0.63 \\
\hline 6 & 3.79 & $3 \cdot 11$ & 0.63 & 0.68 & $0 \cdot 21$ & 0.45 \\
\hline 7 & $12 \cdot 62$ & 9.54 & 0.48 & 0.64 & $0 \cdot 18$ & $0 \cdot 37$ \\
\hline 8 & $4 \cdot 37$ & $3 \cdot 37$ & 0.53 & 0.74 & $0 \cdot 21$ & 0.53 \\
\hline 9 & 9.73 & $7 \cdot 32$ & 0.45 & 0.78 & $0 \cdot 12$ & 0.51 \\
\hline 10 & $4 \cdot 56$ & 3.07 & $0 \cdot 81$ & 0.88 & 0.44 & 0.75 \\
\hline
\end{tabular}




\begin{tabular}{ccr}
\multicolumn{3}{c}{$\begin{array}{c}\text { Table V. Comparison of } \\
\text { ANN3 }\end{array}$} \\
\hline Station & \multicolumn{2}{l}{ CE for validation data } \\
\cline { 2 - 3 } & ANN2 & ANN3 \\
& & \\
\hline 1 & 0.482 & 0.472 \\
2 & 0.587 & 0.577 \\
3 & 0.505 & 0.485 \\
4 & 0.427 & 0.405 \\
5 & 0.573 & 0.557 \\
6 & 0.572 & 0.547 \\
7 & 0.613 & 0.585 \\
8 & 0.473 & 0.470 \\
9 & 0.651 & 0.620 \\
10 & 0.516 & 0.499 \\
\hline
\end{tabular}

\section{SUMMARY AND CONCLUSIONS}

In this paper, an ANN is used to forecast typhoon rainfall. To increase the ability of description, the ANN model developed adopts two hidden layers. The primary objective of this paper is to investigate the effects of the typhoon characteristics and the spatial rainfall information on short-term typhoon-rainfall forecasting. First, the model configuration is evaluated using eight typhoon characteristics. Different models including the lag-1 h, lag- $2 \mathrm{~h}$ and lag- $3 \mathrm{~h}$ are tried to arrive at the optimal model lag. It is found that the lag- $2 \mathrm{~h}$ model has the best performance for each rain gauge. In addition, the forecasts for two testing events (Typhoons Herb and Nelson) show that the model is capable of showing the trend of rainfall when a typhoon is nearby. Second, for improving the model design, the effect of nearby rain gauges on the rainfall forecast at any given location in the study area is considered in the modelling. A semivariogram is also applied to determine the required number of nearby rain gauges whose rainfall information will be used as input to the model. Two testing events (Typhoons Herb and Nelson) support the conclusion that the rainfall can be well forecast by the ANN model when the typhoon characteristics and the appropriate spatial rainfall information are used as inputs to the model. It is also found that too much spatial information cannot improve the generalization ability of the model, because the inclusion of irrelevant information adds noise to the network and undermines the performance of the network. The proposed model is recommended as an alternative to the existing methods, because it considers the influence of both the typhoon characteristics and the spatial rainfall information and requires less time for model development.

\section{ACKNOWLEDGEMENTS}

We are grateful to the Central Weather Bureau and the Water Resources Agency of Taiwan for providing the data. Constructive comments and suggestions from anonymous reviewers are also much appreciated.

\section{REFERENCES}

Burlando P, Rosso R, Cadavid LG, Salas JD. 1993. Forecasting of short-term rainfall using ARMA models. Journal of Hydrology 144: $193-211$.

Eltahir AB. 1998a. A soil moisture-rainfall feedback mechanics: theory and observation. Water Resources Research 34(4): 756-776.

Eltahir AB. 1998b. A soil moisture-rainfall feedback mechanics: numerical experiment. Water Resources Research 34(4): 777-785.

French MN, Krajewsi WF, Cuykendall RR. 1992. Rainfall forecasting in space and time using a neural network. Journal of Hydrology 137: $1-31$.

Georgakakos KP, Bras RL. 1984a. A hydrologically useful station precipitation model: 1. Formulation. Water Resources Research 20(11): $1585-1596$. 
Georgakakos KP, Bras RL. 1984b. A hydrologically useful station precipitation model: 2. Case studies. Water Resources Research 20(11): $1597-1610$.

Govindaraju RS, Rao AR. 2000. Artificial Neural Networks in Hydrology. Kluwer. The Netherlands.

Hsu KL, Gupta V, Sorooshian S. 1995. Artificial neural network modeling of the rainfall-runoff process. Water Resources Research 31(10): $2517-2530$.

Johnson ER, Bras RL. 1980. Multivariate short-term rainfall prediction. Water Resources Research 16(1): $173-185$.

Kitanidis PK. 1993. Geostatistics. In Handbook of Hydrology, Maidment DR (ed.). McGraw-Hill: New York; Chapter 20.

Luk KC, Ball JE, Sharma A. 2000. A study of optimal model lag and spatial inputs to artificial neural network for rainfall forecasting. Journal of Hydrology 227: 56-65.

Minns AW, Hall MJ. 1996. Artificial neural networks as rainfall-runoff models. Hydrological Sciences Journal 41(3): 399-417.

Smith JE, Seo DJ, Baeck ML, Hudlow MD. 1996. An intercomparison study of NEXRAD precipitation estimates. Water Resources Research 32(7): 2035-2045.

Zhu ML, Fujita M. 1994. Comparisons between fuzzy reasoning and neural network methods to forecast runoff discharge. Journal of Hydroscience and Hydraulic Engineering 12(2): 131-141. 